【电子与信息科学／Electronics and Information Science】

\title{
粗䊅集最优近似的动态更新方法
}

\author{
罗来鹏，范自柱 \\ 华东交通大学理学院，江西南昌 330013
}

摘 要：目标概念在近似空间上的近似表示与计算是粗粘集理论模型研究的基础. 对于动态属性约简 及其相关问题往往需要更新粗粘集理论中目标概念的近似性，现有的更新方法都基于目标概念的上、下近 似. 提出一种基于最优近似的动态更新方法，针对新增单个对象引起决策系统中条件类与决策类的动态变 化, 讨论 4 种不同情形下决策类的最优近似动态更新机制, 由此得到一种动态的最优近似集更新算法. 在 UCI 数据集上的测试结果表明, 4 种不同情形下决策类的最优近似更新的时间复杂度存在差异，与非增量 式更新方法相比，所提最优近似粗精集的增量式更新方法可行且高效.

关键词：人工智能；粗䊁集；粒计算；属性约简；最优近似集；增量更新；相似度

中图分类号：TP391 文献标志码：A doi：10.3724/SP. J. 1249.2021.03324

\section{A dynamic approach for updating the optimal approximation of rough set}

\section{LUO Laipeng and FAN Zizhu}

School of Sciences, East China Jiaotong University, Nanchang 330013, Jiangxi Province, P. R. China

\begin{abstract}
The approximate representation and calculation of target concept on approximate space are the foundation of rough set model study. For dynamic attribute reduction and related tasks, it is often necessary to update the approximation of the target concept of rough set theory, and most existing updating approaches are based on the lower and upper approximations of target concepts. This paper presents a dynamic approach for updating the optimal set when a single object is added into the decision table. Aiming at the dynamic change of condition class and decision class caused by the incremental object, the paper discusses the updating mechanisms of optimal approximation set in four different cases and then the dynamic updating algorithm of optimal approximation set is obtained. Finally, the experiments on different data sets from UCI show that the time complexity of optimal approximate updating of decision classes are different for four different cases and the incremental methods are feasible and efficient for decision systems of added one single object.
\end{abstract}

Key words : artificial intelligence; rough set; granular computing; attribute reduction; optimal approximation set; incremental updating; similarity degree

粗糙集理论 ${ }^{[1-2]}$ 是建立在集合论基础上，用来 分析和处理不精确、不协调、不完备信息的数学理 论，是当前 3 大粒计算模型之一，已被成功应用于
人工智能与机器学习等领域. 目标概念在近似空间 上的近似表示与计算，是粗䊁集理论研究与应用的 基础. 当目标概念无法用近似空间上基本知识粒完

Received: 2020-10-29; Accepted: 2021-01-03

Foundation: National Natural Science Foundation of China (61991401); Key Program of Natural Science Foundation of Jiangxi Province (20192ACBL20010)

Corresponding author: Associate professor LUO Laipeng. E-mail: luolp789@163.com

Citation: LUO Laipeng, FAN Zizhu. A dynamic approach for updating the optimal approximation of rough set [J]. Journal of Shenzhen University Science and Engineering, 2021, 38(3) : 324-330. (in Chinese) 
全定义时,一直以来都是借用拓扑中的内核和闭包 概念，采用其上近似集和下近似集作为近似边界来 刻画, 因此, 目标概念在近似空间上往往存在边界 域的不确定性，进而造成系统及规则知识的不确定 性. 为深人挖掘及有效利用边界域信息, ZIARKO $^{[3-4]}$ 提出变精度近似并建立变精度粗糙集模 型和概率粗糙集模型，所得约简规则提高了容错 性, 且能将小样本数据得到的结论用到大数据中. 但是, 这两个模型在实际应用中存在如何优化参数 阈值的问题，参数值直接影响近似集计算和属性约 简结果 ${ }^{[5]}$. 同时, 这种近似的思路仍然是从基本知 识粒即等价类角度来定义目标概念的近似，并未从 系统角度来看待目标概念的近似，且当目标概念是 粗糙时，其近似表示仍是用它的下(上)近似集，而 非一个具体的可定义的集合. 为此, 张清华等 ${ }^{[6-7]}$ 以相似度为度量标准提出目标概念的最优近似, 即 在近似空间上所有可定义的集合中找一个与目标概 念相似度最高的集合作为目标概念的近似. 基于 此, LUO 等 ${ }^{[8]}$ 给出了粗粘集最优近似的代数定义、 计算和基于最优近似的分布约简，讨论了在相同近 似空间下目标概念的最优近似与其上 (下) 近似之 间，以及基于最优近似的分布约简与基于上(下)近 似分布约简之间的关系，发现基于最优近似所建立 的分布约简更能反映数据本身特性，也更能体现粗 粘集应用中不需要先验知识的优势.

然而, 实际应用中数据库中的数据往往是动态 变化的，因此，粗粘集的动态知识发现是目前粗鋉 集研究的重点. 当前研究主要集中在论域变化、属 性集变化和属性值域变化等方面. $\mathrm{YU}$ 等 ${ }^{[9]}$ 针对属 性集变化的区间值信息系统设计了两种动态近似计 算算法; $\mathrm{LI}$ 等 ${ }^{[10]}$ 针对基于优势关系的动态信息系统 讨论了基于对象集的变化时近似集的增量式更新方 法. CHENG 等 ${ }^{[11]}$ 在模糊粗龯集中，提出了两种概 念近似增量更新的动态方法. $\mathrm{HU}$ 等 ${ }^{[12]}$ 在多粒度粗 粘集中，针对单个粒结构随时间演化，提出基于矩 阵的动态近似更新方法. JING 等 ${ }^{[13]}$ 通过引人知识 粒度增量机制，讨论了多个对象发生变化时，更新 约简的两种增量方法. ZHANG 等 ${ }^{[14]}$ 研究了基于知 识粒度的增量属性约简方法. WEI 等 ${ }^{[15]}$ 通过压缩 决策表, 讨论了对象集变化时动态数据的属性约 简. GE 等 ${ }^{[16]}$ 讨论了基于冲突区域的增量机制，提 出在新增对象情况下属性约简的更新计算方法. $\mathrm{HU}$ 等 ${ }^{[17-18]}$ 就多种模型，如优势关系粗糙集模型、 集值粗粘集模型以及不完备系统等，讨论了近似更
新等.

但是，当前关于粗䊁集动态知识获取的研究更 多地局限在基于不同模型目标概念的上、下近似， 随着粗糙集最优近似集的提出，基于粗粘集的最优 近似动态更新计算成为新的研究方向，但目前尚未 见文献报道. 为此, 本研究针对对象集增加的情 况，探讨了决策系统中各决策类的最优近似的动态 变化规律, 为建立基于最优近似的动态分布约简提 供参考.

\section{1 相关概念}

定义 $1^{[1-2]}$ 设 $U$ 是有限论域, $R$ 是 $U$ 上的等价 关系，由此导出近似空间为 $(U, R)$, 等价关系 $R$ 决 定论域 $U$ 上的一种划分称为近似空间 $(U, R)$ 上的 基本知识基，表示为 $U / R=\left\{X_{1}, X_{2}, \cdots, X_{m}\right\}$. 其中, $X_{i} \cap X_{j}=\varnothing(i \neq j), \bigcup_{i=1}^{m} X_{i}=U$, 特别地, 若 $x \in X$ $(i=1,2, \cdots, m)$, 则可用 $[x]_{R}$ 表示 $X_{i}$, 即 $[x]_{R}=X_{i}$.

定义 $2^{[1-2]}$ 设 $(U, R)$ 为近似空间, 且 $U / R=$ $\left\{X_{1}, X_{2}, \cdots, X_{m}\right\}$, 对于 $X \subseteq U$ 的目标概念, 则 $\underline{R}(X)=\left\{x_{p} \mid\left[x_{p}\right]_{R} \subseteq X,\left[x_{p}\right]_{R} \in U / R\right\}$ 和 $\bar{R}(X)=$ $\left\{x_{q} \mid\left[x_{q}\right]_{R} \cap X \neq \varnothing,\left[x_{q}\right]_{R} \in U / R\right\}$ 分别称为 $X$ 在 近似空间 $(U, R)$ 上的下近似集和上近似集, $\operatorname{BN}_{R}(X)=\left\{x_{k} \mid x_{k} \in \bar{R}(X)-\underline{R}(X)\right\}$ 和 $\operatorname{NEG}_{R}(X)=$ $U-\bar{R}(X)$ 分别称为 $X$ 在近似空间 $(U, R)$ 上的边界 域和负域.

定义 $3^{[1-2]}$ 设 $(U, R)$ 为近似空间, 若 $R(X)=$ $\bar{R}(X)$, 则称 $X$ 在近似空间 $(U, R)$ 上是可被定义的 精确集; 否则, 为粗粘集,并用 $(\underline{R}(X), \bar{R}(X))$ 表示.

定义 4 设 $(U, R)$ 为近似空间, $U / R=\left\{X_{1}\right.$, $\left.X_{2}, \cdots, X_{m}\right\}, X \subseteq U$, 则称 $\mathrm{BN}_{R}^{\prime}(X)=$ $\left\{X^{(k)} \mid X^{(k)} \in U / R\right.$ 且 $\left.0<\frac{\left|X^{(k)} \cap X\right|}{\left|X^{(k)}\right|}<1\right\}$ 为 $X$ 的 边界类. 其中, $|\cdot|$ 为集合基数 (下文相同).

定义 5 设 $(U, R)$ 为近似空间, $X \subseteq U$, 在 $(U$, $R)$ 上, 若存在可定义的精确集 $X^{\mathrm{opt}}$, 使得对于任意 可定义的精确集 $Y$, 有 $S\left(X, X^{\mathrm{opt}}\right) \geqslant S(X, Y)$, 则称 $X^{\mathrm{opt}}$ 为 $X$ 在近似空间 $(U, R)$ 上的最优近似集. 其中, $S$ 为 $U$ 上的集合相似度. 对于 $X, Y \subseteq U$, 取 $S(X, Y)=$ $\frac{|X \cap Y|}{|X \cup Y|}$.

定义 6 设 $(U, R)$ 为近似空间, $U / R=\left\{X_{1}\right.$, 
$\left.X_{2}, \cdots, X_{m}\right\}, X \subseteq U$ 的边界域类 $\mathrm{BN}_{R}^{\prime}(X)=\left\{X^{(1)}\right.$, $\left.X^{(2)}, \cdots, X^{(k)}\right\}$, 令 $\frac{\left|X^{(j)} \cap X\right|}{\left|X^{(j)}-X\right|}=\lambda_{j}(j \leqslant k)$, 则称 $\left(\lambda_{1}, \lambda_{2}, \cdots, \lambda_{k}\right)$ 为 $X$ 在 $(U, R)$ 上关于 $\mathrm{BN}_{R}^{\prime}(X)$ 的 分布向量, $\lambda_{j}(j \leqslant k)$ 为对应边界类的分布值.

定理 $\mathbf{1}^{[8]}$ 设 $(U, R)$ 为近似空间, $X \subseteq U$, $\operatorname{BN}_{R}^{\prime}(X)=\left\{X^{(1)}, X^{(2)}, \cdots, X^{(k)}\right\}$. 令 $\frac{|\underline{R}(X)|}{|X|}=\lambda$, $g_{X}\left(\lambda_{j}\right)=\left\{X^{(j)} \mid \frac{\left|X^{(j)} \cap X\right|}{\left|X^{(j)}-X\right|}=\lambda_{j}, X^{(j)} \in \mathrm{BN}_{R}^{\prime}(X)\right\}$, 则 $X$ 在近似空间 $(U, R)$ 上的最优近似集为 $X^{\mathrm{opt}}=$ $\underline{R}(X) \cup \cup_{\lambda_{j} \geqslant \lambda} g_{X}\left(\lambda_{j}\right)$.

由定理 1 可知，计算目标概念的最优近似集的 关键是需要获取两个参数值, 即目标概念中正域所 占的比值和目标概念在其边界类上的分布向量.

\section{2 最优近似集更新原理与算法}

\section{1 最优近似集的更新原理}

更新计算一直都是信息处理研究的重点和难 点. 在粗鉌集动态知识更新内容中, 对象增加是普 遍情况. 考虑到增加多个对象的更新操作可看成是 对增加单个对象更新的递推, 并且对象删除处理方 法具有一定的类似性，接下来将讨论单个对象增加 的情况下，决策系统中决策类的最优近似更新原 理，并设计相应的算法.

设决策系统 $S=(U, C \cup d, V, f)$, 条件属性集 $C$ 在论域 $U$ 上导出的划分为 $U / C=\left\{X_{1}, X_{2}, \cdots\right.$, $\left.X_{m}\right\}$, 决策属性集 $d$ 在 $U$ 上导出的划分为 $U / d=$ $\left\{Y_{1}, Y_{2}, \cdots, Y_{n}\right\}, V$ 为属性值域，并用 $V_{C}$ 表示属性 集 $C$ 的值域, $f$ 为信息函数. 并令 $\lambda_{j}=\frac{\left|\underline{G}\left(Y_{j}\right)\right|}{\left|Y_{j}\right|}$ $(j \leqslant n), Y_{j}$ 关于 $(U, C)$ 在其 $\mathrm{BN}_{C}^{\prime}\left(Y_{j}\right)$ 上的分布向 量 $\boldsymbol{D}_{j}=\left(\lambda_{j}^{(1)}, \lambda_{j}^{(2)}, \cdots, \lambda_{j}^{\left(q_{j}\right)}\right)(j \leqslant n), g_{Y_{j}}\left(\lambda_{i}\right)=$ $\left\{X_{j}^{(i)} \mid \frac{\left|X_{j}^{(i)} \cap Y_{j}\right|}{\left|X_{j}^{(i)}-Y_{j}\right|}=\lambda_{i}, X_{j}^{(i)} \in \operatorname{BN}_{C}^{\prime}\left(Y_{j}\right)\right\}(j \leqslant n)$. 为描述方便，当新增对象 $x$ 后，决策系统 $S$ 改记为 $S^{\prime}=\left(U^{\prime}, C \cup d, V^{\prime}, f\right)$, 相应的 $X_{i}$ 改记为 $X_{i}^{\prime}(i \leqslant m)$, $Y_{j}$ 改记为 $Y_{j}^{\prime}(j \leqslant n), \lambda_{j}$ 改记为 $\lambda_{j}^{\prime}, \boldsymbol{D}_{j}$ 改记为 $\boldsymbol{D}_{j}^{\prime}=$ $\left(\lambda_{j}^{\prime(1)}, \lambda_{j}^{\prime(2)}, \cdots, \lambda_{j}^{\prime\left(q_{j}\right)}\right)(j \leqslant n), V$ 改记为 $V^{\prime}$, 并用 $\lambda_{j}^{(x)}$ 表示 $Y_{j}^{\prime}$ 在新增对象 $x$ 加人的条件类后所对应的 分布值, 新增条件类表示为 $x_{C}$, 新增决策类表示为 $x_{d}$. 此时, 显然有 $U^{\prime}=U \cup x, V^{\prime}=V$ 或 $V \subset V^{\prime}$.
接下来针对对象集新增一个对象并引起值域发 生不同变化的情况，讨论决策类最优近似更新.

定理 2 若 $V_{d}^{\prime}=V_{d}, V_{C}^{\prime} \neq V_{C}$, 且存在 $Y_{j} \in$ $U / d$, 使得 $Y_{j}^{\prime}=Y_{j} \cup x$, 则对于任意 $Y_{k}^{\prime}(k \neq j)$, 有 $\left(Y_{k}^{\prime}\right)^{\mathrm{opt}}=Y_{k}^{\mathrm{opt}},\left(Y_{j}^{\prime}\right)^{\mathrm{opt}}=\left(Y_{j} \cup x\right)^{\mathrm{opt}}=\left\{\underline{C}\left(Y_{j}\right) \cup\right.$ $\{x\}\} \cup \cup_{\lambda \geqslant \lambda_{j}^{\prime}} g_{Y_{j}}(\lambda)$. 其中, $\lambda_{j}^{\prime}=\frac{\left|\underline{C}\left(Y_{j}\right)\right|+1}{\left|Y_{j}\right|+1}$.

【证】因为 $V_{d}^{\prime}=V_{d}, V_{C}^{\prime} \neq V_{C}$, 所以 $f(x, d) \in$ $V_{d}, f(x, C) \notin V_{C}$, 又因为 $Y_{j}^{\prime}=Y_{j} \cup x$, 所以 $U^{\prime} / C=$ $\left\{X_{1}^{\prime}, X_{2}^{\prime}, \cdots, X_{m}^{\prime}, x_{C}\right\}, U^{\prime} / d=\left\{Y_{1}^{\prime}, Y_{2}^{\prime}, \cdots, Y_{j}^{\prime}, \cdots\right.$, $\left.Y_{n}^{\prime}\right\}$. 其中, $X_{i}^{\prime}=X_{i}(i \leqslant m), Y_{k}^{\prime}=Y_{k}(k \neq j, k \leqslant$ $n)$. 此时, 任意 $Y_{k}^{\prime}(k \neq j)$ 与 $Y_{k}$ 具有相同边界分布且 正域不变, 所以 $\left(Y_{k}^{\prime}\right)^{\mathrm{opt}}=Y_{k}^{\mathrm{opt}}$. 而对于 $Y_{j}^{\prime}=Y_{j} \cup x$, 由 于 $\underline{C}\left(Y_{j}^{\prime}\right)=\underline{C}\left(Y_{j} \cup\{x\}\right)=\underline{C}\left(Y_{j}\right) \cup\{x\}$, 所以 $\lambda_{j}^{\prime}=$ $\frac{\left|\underline{G}\left(Y_{j}\right)\right|+1}{\left|Y_{j}\right|+1}$. 又因对于任意 $i(i \leqslant m)$, 有 $\frac{\left|X_{i}^{\prime} \cap Y_{j}^{\prime}\right|}{\left|X_{i}^{\prime}-Y_{j}^{\prime}\right|}=$ $\frac{\left|X_{i} \cap Y_{j}\right|}{\left|X_{i}-Y_{j}\right|}$, 即 $Y_{j}$ 和 $Y_{j}^{\prime}$ 关于 $(U, C)$ 在其边界类上的 分布值相同, 所以 $\left(Y_{j}^{\prime}\right)^{\mathrm{opt}}=\left(Y_{j} \cup x\right)^{\mathrm{opt}}=\left\{\underline{C}\left(Y_{j}\right) \cup\right.$ $\{x\}\} \cup \underset{\lambda \geqslant \lambda_{j}^{\prime}}{\cup} g_{Y_{j}}(\lambda)$.

定理 3 若 $V_{d}^{\prime}=V_{d}, V_{c}^{\prime}=V_{C}$, 且存在 $Y_{j} \in$ $U / d$, 使得 $Y_{j}^{\prime}=Y_{j} \cup x$, 存在 $X_{i} \in U / C, X_{i}^{\prime}=X_{i} \cup$ $x$, 则对于任意 $Y_{k}^{\prime}(k \neq j)$, 有:

1) 若 $Y_{k} \cap X_{i}=\varnothing$, 则 $\left(Y_{k}^{\prime}\right)^{\mathrm{opt}}=Y_{k}^{\mathrm{opt}}$;

2) 若 $X_{i} \subseteq \underline{C}\left(Y_{k}\right)$, 则 $\left(Y_{k}^{\prime}\right)^{\text {opt }}=\{x\} \cup \underline{C}\left(Y_{k}\right) \cup$ $\bigcup_{\lambda \geqslant \lambda_{k}^{\prime}} g_{Y_{k}}(\lambda)$. 其中, $\lambda_{k}^{\prime}=\frac{\left|\underline{C}\left(Y_{k}\right)-X_{i}\right|}{\left|Y_{k}\right|}$;

3) 若 $X_{i} \in \mathrm{BN}_{C}^{\prime}\left(Y_{k}\right)$, 则当 $\frac{\left|X_{i} \cap Y_{k}\right|}{\left|X_{i}-Y_{k}\right|+1} \geqslant \lambda_{k}^{\prime}$ 时, $\left(Y_{k}^{\prime}\right)^{\text {opt }}=Y_{k}^{\text {opt }} \cup x$; 当 $\lambda_{k}^{\prime} \geqslant \frac{\left|X_{i} \cap Y_{k}\right|}{\left|X_{i}-Y_{k}\right|}$ 时, $\left(Y_{k}^{\prime}\right)^{\mathrm{opt}}=Y_{k}^{\mathrm{opt}}$; 当 $\frac{\left|X_{i} \cap Y_{k}\right|}{\left|X_{i}-Y_{k}\right|} \geqslant \lambda_{k}^{\prime}>\frac{\left|X_{i} \cap Y_{k}\right|}{\left|X_{i}-Y_{k}\right|+1}$ 时, $\left(Y_{k}^{\prime}\right)^{\mathrm{opt}}=Y_{k}^{\mathrm{opt}}-X_{i}$. 其中, $\lambda_{k}^{\prime}=\frac{\left|\underline{G}\left(Y_{k}\right)\right|}{\left|Y_{k}\right|}$.

【证】由于 $V_{d}^{\prime}=V_{d}, V_{C}^{\prime}=V_{C}$, 且 $Y_{j}^{\prime}=Y_{j} \cup x$, $X_{i}^{\prime}=X_{i} \cup x$, 即 $U^{\prime} / C=\left\{X_{1}^{\prime}, X_{2}^{\prime}, \cdots, X_{i} \cup x, \cdots\right.$, $\left.X_{m}{ }^{\prime}\right\}, U^{\prime} / d=\left\{Y_{1}^{\prime}, Y_{2}^{\prime}, \cdots, Y_{j} \cup x, \cdots, Y_{n}^{\prime}\right\}$. 其中, $X_{p}^{\prime}=X_{p}(p \neq i, p \leqslant m), Y_{t}^{\prime}=Y_{t}(t \neq j, t \leqslant n)$. 因 此, 有:

1) 对于任意 $k \neq j$, 若 $Y_{k} \cap X_{i}=\varnothing$, 则 $Y_{k}^{\prime} \cap$ $X_{i}^{\prime}=\varnothing$, 所以 $Y_{k}^{\prime}(k \neq j)$ 与 $Y_{k}$ 具有相同边界分布且 
正域不变, 所以 $\left(Y_{k}^{\prime}\right)^{\text {opt }}=Y_{k}^{\text {opt }}$.

2) 若 $X_{i} \subseteq \underline{C}\left(Y_{k}\right)(k \neq j, k \leqslant n)$, 因为 $x \notin Y_{k}$, 所以 $\underline{C}\left(Y_{k}^{\prime}\right)=\underline{C}\left(Y_{k}\right)-X_{i}$, 且类 $X_{i}^{\prime}=X_{i} \cup\{x\}$ 变成 $\mathrm{BN}_{C}^{\prime}\left(Y_{k}^{\prime}\right)$ 中的新元素. 此时, $\lambda_{k}^{\prime}=\frac{\left|\underline{C}\left(Y_{k}\right)-X_{i}\right|}{\left|Y_{k}\right|}$, 而 新增的边界类 $X_{i} \cup\{x\}$ 的分布值 $\lambda_{k}^{(x)}=$ $\frac{\left|\left(X_{i} \cup\{x\}\right) \cap Y_{k}^{\prime}\right|}{\left|\left(X_{i} \cup\{x\}\right)-Y_{k}^{\prime}\right|}=\frac{\left|X_{i} \cap Y_{k}\right|}{|\{x\}|}=\left|X_{i} \cap Y_{k}\right|=$ $\left|X_{i}\right| \geqslant 1 \geqslant \lambda_{k}^{\prime}$, 因此, $Y_{k}^{\prime}$ 的最优近似集 $\left(Y_{k}^{\prime}\right)^{\mathrm{opt}}=$ $\{x\} \cup \underline{C}\left(Y_{k}\right) \cup \cup_{\lambda \geqslant \lambda_{k}^{\prime}} g_{Y_{k}}(\lambda)$.

3) 若 $X_{i} \in \mathrm{BN}_{C}^{\prime}\left(Y_{k}\right)$, 则 $Y_{k}$ 和 $Y_{k}^{\prime}$ 的正域不变, 即 $\lambda_{k}^{\prime}=\lambda_{k}=\frac{\left|\underline{G}\left(Y_{k}\right)\right|}{\left|Y_{k}\right|}$. 此时, $Y_{k}$ 和 $Y_{k}^{\prime}$ 在边界域分布 中唯有 $X_{i}$ 所对应的 $X_{i}^{\prime}$ 分布值发生改变, 且值为

$$
\begin{aligned}
\lambda_{k}^{(x)}= & \frac{\left|X_{i}^{\prime} \cap Y_{k}^{\prime}\right|}{\left|X_{i}^{\prime}-Y_{k}^{\prime}\right|}=\frac{\left|\left(X_{i} \cup\{x\}\right) \cap Y_{k}^{\prime}\right|}{\left|\left(X_{i} \cup\{x\}\right)-Y_{k}^{\prime}\right|}= \\
& \frac{\left|X_{i} \cap Y_{k}\right|}{\left|X_{i}-Y_{k}\right|+1}<\frac{\left|X_{i} \cap Y_{k}\right|}{\left|X_{i}-Y_{k}\right|} .
\end{aligned}
$$

因此, 若 $\lambda_{k}^{(x)} \geqslant \lambda_{k}^{\prime}, X_{i}$ 增加 $x$ 后归属的最优近 似保持不变, 其他分布也未改变, 则有 $\left(Y_{k}^{\prime}\right)^{\mathrm{opt}}=$ $Y_{k}^{\mathrm{opt}} \cup\{x\}$. 类似地, 当 $\lambda_{k}^{\prime}>\frac{\left|X_{i} \cap Y_{k}\right|}{\left|X_{i}-Y_{k}\right|},\left(Y_{k}^{\prime}\right)^{\mathrm{opt}}=$ $Y_{k}^{\text {opt }}$; 当 $\frac{\left|X_{i} \cap Y_{k}\right|}{\left|X_{i}-Y_{k}\right|} \geqslant \lambda_{k}^{\prime}>\frac{\left|X_{i} \cap Y_{k}\right|}{\left|X_{i}-Y_{k}\right|+1}$ 时, $\left(Y_{k}^{\prime}\right)^{\mathrm{opt}}=\left(Y_{k}\right)^{\mathrm{opt}}-X_{i}$.

定理 4 若 $V_{d}^{\prime}=V_{d}, V_{C}^{\prime}=V_{C}$, 且存在 $Y_{j} \in U / d$ 使得 $Y_{j}^{\prime}=Y_{j} \cup x$, 存在 $X_{i} \in U / C$ 使得 $X_{i}^{\prime}=X_{i} \cup x$, 则

1) 若 $X_{i} \subseteq \underline{C}\left(Y_{j}\right)$, 则 $\left(Y_{j}^{\prime}\right)^{\mathrm{opt}}=\{x\} \cup \underline{C}\left(Y_{j}\right) \cup$ $\bigcup_{\lambda \geqslant \lambda_{j}} g_{Y_{j}}(\lambda)$. 其中, $\lambda_{j}^{\prime}=\frac{\left|\underline{G}\left(Y_{j}\right)\right|+1}{\left|Y_{j}\right|+1}$.

2) 若 $X_{i} \cap Y_{j}=\varnothing$, 则当 $\frac{1}{\left|X_{i}\right|} \geqslant \lambda_{j}^{\prime}$ 时, $\left(Y_{j}^{\prime}\right)^{\mathrm{opt}}=$ $\underline{C}\left(Y_{j}\right) \cup \cup_{\lambda \geqslant \lambda_{k}^{\prime}} g_{Y_{j}}(\lambda) \cup X_{i} \cup\{x\}$; 当 $\frac{1}{\left|X_{i}\right|}<\lambda_{j}^{\prime}$ 时, $\left(Y_{j}^{\prime}\right)^{\text {opt }}=\underline{C}\left(Y_{j}\right) \cup \cup_{\lambda \geqslant \lambda_{j}^{\prime}} g_{Y_{j}}(\lambda)$. 其中, $\lambda_{j}^{\prime}=$ $\frac{\left|\underline{C}\left(Y_{j}\right)\right|}{\left|Y_{j}\right|+1}$.

$3)$ 若 $X_{i} \in \mathrm{BN}_{C}^{\prime}\left(Y_{j}\right)$, 则当 $\lambda_{j}^{(x)} \geqslant \lambda_{j}^{\prime}$ 时, $\left(Y_{j}^{\prime}\right)^{\mathrm{opt}}=$ $X_{i} \cup\{x\} \cup \underline{C}\left(Y_{j}\right) \cup \cup_{\lambda \geqslant \lambda_{j}} g_{Y_{j}}(\lambda)$; 当 $\lambda_{j}^{(x)}<\lambda_{j}^{\prime}$ 时, $\left(Y_{j}^{\prime}\right)^{\mathrm{opt}}=\underline{C}\left(Y_{j}\right) \cup \cup_{\lambda \geqslant \lambda_{j}} g_{Y_{j}}(\lambda)-X_{i}$. 其中, $\lambda_{j}^{\prime}=$
$\frac{\left|\underline{C}\left(Y_{j}\right)\right|}{\left|Y_{j}\right|+1}, \lambda_{j}^{(x)}=\frac{\left|X_{i} \cap Y_{j}\right|+1}{\left|X_{i}-Y_{j}\right|}$.

【证】

1) 因为 $X_{i} \subseteq \underline{C}\left(Y_{j}\right)$, 且 $X_{i}^{\prime}=X_{i} \cup x, Y_{j}^{\prime}=$ $Y_{j} \cup x$, 所以 $X_{i}^{\prime} \subseteq \underline{G}\left(Y_{j}^{\prime}\right), \lambda_{j}^{\prime}=\frac{\left|\underline{G}\left(Y_{j}^{\prime}\right)\right|}{\left|Y_{j}^{\prime}\right|}=$ $\frac{\left|\underline{G}\left(Y_{j}\right)\right|+1}{\left|Y_{j}\right|+1}$. 又因 $Y_{j}$ 与 $Y_{j}^{\prime}$ 的边界类及分布值不变, 所以 $\left(Y_{j}^{\prime}\right)^{\text {opt }}=\{x\} \cup \underline{C}\left(Y_{j}\right) \cup \bigcup_{\lambda \geqslant \lambda_{j}} g_{Y_{j}}(\lambda)$.

2) 若 $X_{i} \cap Y_{j}=\varnothing$, 则 $X_{i}^{\prime} \in \mathrm{BN}_{C}\left(Y_{j}^{\prime}\right), Y_{j}^{\prime}$ 比 $Y_{j}$ 仅新增一个边界类 $X_{i}^{\prime}$, 并且 $\lambda_{j}^{\prime}=\frac{\left|\underline{G}\left(Y_{j}\right)\right|}{\left|Y_{j}\right|+1}$, 而 $\lambda_{j}^{(x)}=\frac{|\{x\}|}{\left|X_{i}\right|}=\frac{1}{\left|X_{i}\right|}, Y_{j}$ 与 $Y_{j}^{\prime}$ 的其他的边界类和 它们对应的分布值不变, 所以当 $\frac{1}{\left|X_{i}\right|} \geqslant \lambda_{j}^{\prime}$ 时, $\left(Y_{j}^{\prime}\right)^{\text {opt }}=\underline{C}\left(Y_{j}\right) \cup \underset{\lambda \geqslant \lambda_{k}^{\prime}}{\cup} g_{Y_{j}}(\lambda) \cup X_{i} \cup\{x\}$; 当 $\frac{1}{\left|X_{i}\right|}<$ $\lambda_{j}^{\prime}$ 时, $\left(Y_{j}^{\prime}\right)^{\mathrm{opt}}=\underline{C}\left(Y_{j}\right) \cup \bigcup_{\lambda \geqslant \lambda_{j}^{\prime}} g_{Y_{j}}(\lambda)$

$3)$ 若 $X_{i} \in \mathrm{BN}_{C}^{\prime}\left(Y_{j}\right)$, 则 $\underline{C}\left(Y_{j}\right)=\underline{G}\left(Y_{j}^{\prime}\right), \lambda_{j}^{\prime}=$ $\frac{\left|\underline{G}\left(Y_{j}\right)\right|}{\left|Y_{j}\right|+1}, Y_{j}^{\prime}$ 在其所有边界类中唯有在 $X_{i}^{\prime}$ 上的分布 值发生改变, $\lambda_{j}^{(x)}=\frac{\left|X_{i}^{\prime} \cap Y_{j}^{\prime}\right|}{\left|X_{i}^{\prime}-Y_{j}^{\prime}\right|}=\frac{\left|X_{i} \cap Y_{j}\right|+1}{\left|X_{i}-Y_{j}\right|}$. 所 以, 若 $\lambda_{j}^{(x)} \geqslant \lambda_{j}^{\prime}$, 则 $\left(Y_{j}^{\prime}\right)^{\mathrm{opt}}=X_{i} \cup\{x\} \cup \underline{C}\left(Y_{j}\right) \cup$ $\bigcup_{\lambda \geqslant \lambda_{j}^{\prime}} g_{Y_{j}}(\lambda)$; 若 $\lambda_{j}^{(x)}<\lambda_{j}^{\prime}$, 则 $\left(Y_{j}^{\prime}\right)^{\text {opt }}=\underline{C}\left(Y_{j}\right) \cup$ $\cup_{\lambda \geqslant \lambda_{j}^{\prime}} g_{Y_{j}}(\lambda)-X_{i}$.

定理 5 若 $V_{d}^{\prime} \neq V_{d}, V_{C}^{\prime} \neq V_{C}$, 则 $\left(Y_{j}^{\prime}\right)^{\mathrm{opt}}=$ $Y_{j}^{\mathrm{opt}}(j \leqslant n), x_{d}^{\mathrm{opt}}=x_{C}$.

【证】若 $V_{d}^{\prime} \neq V_{d}$ 且 $V_{c}^{\prime} \neq V_{C}$, 则 $U^{\prime} / C=\left\{X_{1}^{\prime}\right.$, $\left.X_{2}^{\prime}, \cdots, X_{m}^{\prime}, x_{C}\right\}, U^{\prime} / d=\left\{Y_{1}^{\prime}, Y_{2}^{\prime}, \cdots, Y_{n}^{\prime}, x_{d}\right\}$, 且 $X_{i}^{\prime}=X_{i}(i \leqslant m), Y_{j}^{\prime}=Y_{j}(j \leqslant n)$. 此时显然有 $\left(Y_{j}^{\prime}\right)^{\mathrm{opt}}=Y_{j}^{\mathrm{opt}}(j \leqslant n), x_{d}^{\mathrm{opt}}=x_{C}$.

定理 6 若 $V_{d}^{\prime} \neq V_{d}, V_{C}^{\prime}=V_{C}$, 且存在 $X_{i} \in U / C$ 使得 $X_{i}^{\prime}=X_{i} \cup x$, 对于任意 $Y_{j}^{\prime}(j \leqslant n)$, 则有:

1) $x_{d}^{\mathrm{opt}}=X_{i}^{\prime}=X_{i} \cup x$.

2) 若 $X_{i} \subseteq \underline{C}\left(Y_{j}\right)$, 则 $\left(Y_{j}^{\prime}\right)^{\mathrm{opt}}=\{x\} \cup \underline{C}\left(Y_{j}\right) \cup$ $\cup_{\lambda \geqslant \lambda_{j}^{\prime}} g_{Y_{j}}(\lambda)$. 其中, $\lambda_{j}^{\prime}=\frac{\left|\underline{C}\left(Y_{j}\right)-X_{i}\right|}{\left|Y_{j}\right|}$.

3) 若 $X_{i} \in \mathrm{BN}_{C}^{\prime}\left(Y_{j}\right)$, 则当 $\lambda_{j}^{(x)} \geqslant \lambda_{j}$ 时, $\left(Y_{j}^{\prime}\right)^{\mathrm{opt}}=Y_{j}^{\mathrm{opt}} \cup\{x\}$; 当 $\lambda_{j}^{(x)}<\lambda_{j}$ 时, $\left(Y_{j}^{\prime}\right)^{\mathrm{opt}}=Y_{j}^{\mathrm{opt}}-$ 
$X_{i}$. 其中, $\lambda_{j}^{(x)}=\frac{\left|X_{i} \cap Y_{j}\right|}{\left|X_{i}-Y_{j}\right|+1}$.

4) 若 $X_{i} \cap Y_{j}=\varnothing$, 则 $\left(Y_{j}^{\prime}\right)^{\text {opt }}=Y_{j}^{\text {opt }}$.

【证】若 $V_{d}^{\prime} \neq V_{d}, V_{C}^{\prime}=V_{C}$, 且存在 $X_{i} \in U / C$ 使得 $X_{i}^{\prime}=X_{i} \cup x$, 此时有 $U / C=\left\{X_{1}^{\prime}, X_{2}^{\prime}, \cdots\right.$, $\left.X_{i} \cup x, \cdots, X_{m}^{\prime}\right\}, U^{\prime} / d=\left\{Y_{1}^{\prime}, Y_{2}^{\prime}, \cdots, Y_{n}^{\prime}, x_{d}\right\}$, 且 $X_{p}^{\prime}=X_{p}(p \neq i, p \leqslant m), Y_{j}^{\prime}=Y_{j}(j \leqslant n)$, 则

1) $x_{d}^{\mathrm{opt}}=X_{i}^{\prime}=X_{i} \cup x$ 显然成立;

2) 若 $X_{i} \subseteq \underline{C}\left(Y_{j}\right)(j \leqslant n)$, 则类 $X_{i}^{\prime}=X_{i} \cup\{x\}$ 变成 $\mathrm{BN}_{C}^{\prime}\left(Y_{j}^{\prime}\right)$ 中的新元素, 此时 $\lambda_{j}^{\prime}=$ $\frac{\left|\underline{C}\left(Y_{j}\right)-X_{i}\right|}{\left|Y_{j}\right|}, \quad \lambda_{j}^{(x)}=\frac{\left|\left(X_{i} \cup\{x\}\right) \cap Y_{j}^{\prime}\right|}{\left|\left(X_{i} \cup\{x\}\right)-Y_{j}^{\prime}\right|}=$ $\frac{\left|X_{i} \cap Y_{j}\right|}{|\{x\}|}=\left|X_{i}\right| \geqslant 1 \geqslant \lambda_{j}^{\prime}$, 因此 $\left(Y_{j}^{\prime}\right)^{\mathrm{opt}}=\underline{C}\left(Y_{j}\right) \cup$ $\{x\} \cup \bigcup_{\lambda \geqslant \lambda_{j}^{\prime}} g_{Y_{j}}(\lambda)$.

3) 若 $X_{i} \subseteq \mathrm{BN}_{C}^{\prime}\left(Y_{j}\right)$, 则 $\lambda_{j}^{\prime}=\lambda_{j}=\frac{\left|\underline{G}\left(Y_{j}\right)\right|}{\left|Y_{j}\right|}$, $Y_{j}$ 在边界类的分布中唯有 $X_{i}$ 所对应的值发生了改 变, $\lambda_{j}^{(x)}=\frac{\left|\left(X_{i} \cup\{x\}\right) \cap Y_{j}^{\prime}\right|}{\left|\left(X_{i} \cup\{x\}\right)-Y_{j}^{\prime}\right|}=\frac{\left|X_{i} \cap Y_{j}\right|}{\left|X_{i}-Y_{j}\right|+1}$. 因 此, 若 $\lambda_{j}^{(x)} \geqslant \lambda_{j}^{\prime}$, 则 $\left(Y_{j}^{\prime}\right)^{\mathrm{opt}}=Y_{j}^{\mathrm{opt}} \cup\{x\}$; 若 $\lambda_{j}^{(x)}<\lambda_{j}^{\prime}$, 则 $\left(Y_{j}^{\prime}\right)^{\mathrm{opt}}=Y_{j}^{\mathrm{opt}}-X_{i}$.

4) 若 $X_{i} \cap Y_{i}=\varnothing$, 此时显然 $\left(Y_{j}^{\prime}\right)^{\mathrm{opt}}=Y_{j}^{\mathrm{opt}}$.

综上可见, 新增一个对象后, 决策系统中决策 类最优近似更新的计算, 只需分析变化的条件类对 决策类正域、边界域和负域的改变情况. 显然, 与非 增量计算相比, 增量更新计算可极大地简化求解过 程; 同时, 因新增对象所引起的属性值域的变化不 尽相同，决策类更新的时间复杂度也未必一样.

\section{2 最优近似集的更新算法描述}

设决策系统 $S=(U, C \cup d, V, f)$, 条件属性集 $C$ 在论域 $U$ 上导出的划分为 $U / C=\left\{X_{1}, X_{2}, \cdots\right.$, $\left.X_{m}\right\}$, 决策属性集 $d$ 在 $U$ 上导出的划分为 $U / d=$ $\left\{Y_{1}, Y_{2}, \cdots, Y_{n}\right\}$, 并令 $\lambda_{j}=\frac{\left|\underline{G}\left(Y_{j}\right)\right|}{\left|Y_{j}\right|}(j \leqslant n)$, 则 当新增对象 $x$ 后, $X_{i}(i \leqslant m)$ 变为 $X_{i}^{\prime}, Y_{j}(j \leqslant n)$ 变为 $Y_{j}^{\prime}, \lambda_{j}$ 变为 $\lambda_{j}^{\prime}$. 最优近似集更新算法步骤为:

步骤 1 获取相关变量的初始值. 计算 $\underline{C}\left(Y_{j}\right)$ 、 $\lambda_{j}=\frac{\left|\underline{C}\left(Y_{j}\right)\right|}{\left|Y_{j}\right|} 、 \mathrm{BN}_{C}^{\prime}\left(Y_{j}\right) 、 g_{Y_{j}}\left(\lambda_{i}\right)$ 和 $Y_{j}^{\mathrm{opt}}(j \leqslant n)$.

步骤 2 对新增数据类别进行判定，并计算决 策类最优近似的更新结果.

1) 若 $V_{d}^{\prime} \neq V_{d}$ 且 $V_{C}^{\prime} \neq V_{C}$, 即令 $X_{k}^{\prime}=X_{k}(k \leqslant$ $m), Y_{k}^{\prime}=Y_{k}(k \leqslant n)$, 则 $\left(Y_{j}^{\prime}\right)^{\mathrm{opt}}=Y_{j}^{\mathrm{opt}}(j \leqslant n)$, $x_{d}^{\mathrm{opt}}=x_{C}$.

2) 若 $V_{d}^{\prime} \neq V_{d} V_{C}^{\prime}=V_{C}$, 即令 $X_{i}^{\prime}=X_{i} \cup x, X_{k}^{\prime}=$ $X_{k}(k \neq i), Y_{k}^{\prime}=Y_{k}(k \leqslant n)$, 则对于 $Y_{j}^{\prime}(j \leqslant n)$, 有: (1) $x_{d}^{\mathrm{opt}}=X_{i}^{\prime}=X_{i} \cup x$. (2) 若 $X_{i} \subseteq \underline{C}\left(Y_{j}\right)$, 则 $\left(Y_{j}^{\prime}\right)^{\mathrm{opt}}=$ $\{x\} \cup \underline{G}\left(Y_{j}\right) \cup \underset{\lambda \geqslant \lambda_{j}^{\prime}}{\cup} g_{Y_{j}}(\lambda)$. 其中, $\lambda_{j}^{\prime}=$ $\frac{\left|\underline{C}\left(Y_{j}\right)-X_{i}\right|}{\left|Y_{j}\right|}$. (3) 若 $X_{i} \in \mathrm{BN}_{C}^{\prime}\left(Y_{j}\right)$, 则当 $\lambda_{j}^{(x)} \geqslant \lambda_{j}$ 时, 有 $\left(Y_{j}^{\prime}\right)^{\mathrm{opt}}=Y_{j}^{\mathrm{opt}} \cup\{x\}$; 当 $\lambda_{j}^{\prime(x)}<\lambda_{j}$ 时, 有 $\left(Y_{j}^{\prime}\right)^{\mathrm{opt}}=Y_{j}^{\mathrm{opt}}-X_{i}$. 其中, $\lambda_{j}^{\prime(x)}=\frac{\left|X_{i} \cap Y_{j}\right|}{\left|X_{i}-Y_{j}\right|+1}, \lambda_{j}=$ $\frac{\left|\underline{C}\left(Y_{j}\right)\right|}{\left|Y_{j}\right|}$. (4) 若 $X_{i} \cap Y_{j}=\varnothing$, 则 $\left(Y_{j}^{\prime}\right)^{\mathrm{opt}}=Y_{j}^{\mathrm{opt}}$.

$3)$ 若 $V_{d}^{\prime}=V_{d}$ 且 $V_{C}^{\prime} \neq V_{C}$, 即令 $X_{k}^{\prime}=X_{k}(k \leqslant$ $m), Y_{k}^{\prime}=Y_{k}(k \neq j), Y_{j}^{\prime}=Y_{j} \cup x$, 则 $\left(Y_{k}^{\prime}\right)^{\mathrm{opt}}=$ $Y_{k}^{\text {opt }}(k \neq j), \quad\left(Y_{j}^{\prime}\right)^{\text {opt }}=\left\{\underline{C}\left(Y_{j}\right) \cup\{x\}\right\} \cup$ $\bigcup_{\lambda \geqslant \lambda_{j}^{\prime}} g_{Y_{j}}(\lambda)$. 其中, $\lambda_{j}^{\prime}=\frac{\left|\underline{G}\left(Y_{j}\right)\right|+1}{\left|Y_{j}\right|+1}$.

4) 若 $V_{d}^{\prime}=V_{d}$ 且 $V_{C}^{\prime}=V_{C}$, 即令 $Y_{j}^{\prime}=Y_{j} \cup x, X_{i}^{\prime}=$ $X_{i} \cup x, X_{k}^{\prime}=X_{k}(k \neq i), Y_{k}^{\prime}=Y_{k}(k \neq j)$, 对于任意 $Y_{k}^{\prime}$, 有: (1) 若 $Y_{k} \cap X_{i}=\varnothing$, 则 $\left(Y_{k}^{\prime}\right)^{\mathrm{opt}}=Y_{k}^{\mathrm{opt}}(k \neq j)$. 当 $\frac{1}{\left|X_{i}\right|} \geqslant \lambda_{j}^{\prime}$ 时, $\left(Y_{j}^{\prime}\right)^{\mathrm{opt}}=\underline{C}\left(Y_{j}\right) \cup \cup_{\lambda \geqslant \lambda_{k}^{\prime}} g_{Y_{j}}(\lambda) \cup X_{i} \cup$ $\{x\}$; 当 $\frac{1}{\left|X_{i}\right|}<\lambda_{j}^{\prime}$ 时, $\left(Y_{j}^{\prime}\right)^{\mathrm{opt}}=\underline{C}\left(Y_{j}\right) \cup \cup_{\lambda \geqslant \lambda_{k}^{\prime}} g_{Y_{j}}(\lambda)$. 其中, $\lambda_{k}^{\prime}=\frac{\left|\underline{G}\left(Y_{j}\right)\right|}{\left|Y_{j}\right|+1}$. (2) 若 $X_{i} \subseteq \underline{G}\left(Y_{k}\right)$, 则 $\left(Y_{k}^{\prime}\right)^{\mathrm{opt}}=$ $\{x\} \cup \underline{G}\left(Y_{k}\right) \cup \underset{\lambda \geqslant \lambda_{k}^{\prime}}{\cup} g_{Y_{j}}(\lambda)(k \neq j)$. 其中, $\lambda_{k}^{\prime}=$ $\frac{\left|\underline{C}\left(Y_{k}\right)-X_{i}\right|}{\left|Y_{k}\right|} ;\left(Y_{j}^{\prime}\right)^{\mathrm{opt}}=\{x\} \cup \underline{G}\left(Y_{j}\right) \cup \cup_{\lambda \geqslant \lambda_{j}^{\prime}} g_{Y_{j}}(\lambda)$. 其中, $\lambda_{j}^{\prime}=\frac{\left|\underline{G}\left(Y_{j}\right)\right|+1}{\left|Y_{j}\right|+1}$. (3) 若 $X_{i} \in \mathrm{BN}_{C}^{\prime}\left(Y_{k}\right)$, 则 当 $\frac{\left|X_{i} \cap Y_{k}\right|}{\left|X_{i}-Y_{k}\right|+1} \geqslant \lambda_{k}^{\prime}$ 时, $\left(Y_{k}^{\prime}\right)^{\mathrm{opt}}=Y_{k}^{\mathrm{opt}} \cup x(k \neq j)$; 当 $\lambda_{k}^{\prime}>\frac{\left|X_{i} \cap Y_{k}\right|}{\left|X_{i}-Y_{k}\right|}$ 时, $\left(Y_{k}^{\prime}\right)^{\mathrm{opt}}=Y_{k}^{\mathrm{opt}}(k \neq j)$; 当 $\frac{\left|X_{i} \cap Y_{k}\right|}{\left|X_{i}-Y_{k}\right|} \geqslant \lambda_{k}^{\prime}>\frac{\left|X_{i} \cap Y_{k}\right|}{\left|X_{i}-Y_{k}\right|+1}$ 时, $\left(Y_{k}^{\prime}\right)^{\mathrm{opt}}=Y_{k}^{\mathrm{opt}}-$ $X_{i}(k \neq j)$, 其中, $\lambda_{j}^{\prime}=\frac{\left|\underline{C}\left(Y_{k}\right)\right|}{\left|Y_{k}\right|}$; 当 $\lambda_{j}^{(x)} \geqslant \lambda_{j}^{\prime}$ 时, $\left(Y_{j}^{\prime}\right)^{\mathrm{opt}}=X_{i} \cup\{x\} \cup \underline{C}\left(Y_{j}\right) \cup \cup_{\lambda \geqslant \lambda_{j}^{\prime}} g_{Y_{j}}(\lambda)$; 当 $\lambda_{j}^{(x)}<\lambda_{j}^{\prime}$ 时, $\left(Y_{j}^{\prime}\right)^{\mathrm{opt}}=\underline{C}\left(Y_{j}\right) \cup \cup_{\lambda \geqslant \lambda_{j}^{\prime}} g_{Y_{j}}(\lambda)-X_{i}$. 其中, $\lambda_{j}^{\prime}=$ 
$\frac{\left|\underline{C}\left(Y_{j}\right)\right|}{\left|Y_{j}\right|+1} ; \lambda_{j}^{(x)}=\frac{\left|X_{i} \cap Y_{j}\right|+1}{\left|X_{i}-Y_{j}\right|}$.

\section{3 实验方案及性能比较}

\section{1 实验方案}

从 UCI 公用数据集 (http://archive. ics. uci. edu/ml/index. php) 选择数据集 Zoo、Hayes-roth、 Lymphography、Monk's problems 和 Balance-scale, 采 用 SQL server 2008 中的 SQL 进行编程, 测试不同条 件下最优近似更新计算的运行时间. 实验测试所用 的计算机配置为: 内存为 8 Gbyte, CPU 为 Intel $^{\circledR}$ Core (TM) i7-8550U, $1.80 \mathrm{GHz}$, 操作系统为 Windows 10. 表 1 为 5 个数据集的参数描述.

\section{表 1 数据集描述}

Table 1 Data sets

\begin{tabular}{ccccc}
\hline 数据集 & $\begin{array}{c}\text { 对象 } \\
\text { 数/个 }\end{array}$ & $\begin{array}{c}\text { 属性 } \\
\text { 数/个 }\end{array}$ & $\begin{array}{c}\text { 决策 } \\
\text { 类数/个 }\end{array}$ & 缺失值 \\
\hline Zoo & 101 & 17 & 7 & 无 \\
Hayes-roth & 132 & 5 & 3 & 无 \\
Lymphography & 148 & 18 & 4 & 无 \\
Monk's problems & 432 & 7 & 2 & 无 \\
Balance-scale & 625 & 4 & 3 & 无 \\
\hline
\end{tabular}

\section{2 结果与分析}

图 1 为在 5 个数据集上新增不同类别的单个对 象时, 最优近似更新计算的运行时间对比. 其中, 每种情况下的运行时间取相同类别数据 5 次运行时 间的平均值. 由图 1 可见, 满足条件 $V_{d}^{\prime} \neq V_{d}$ 且 $V_{C}^{\prime} \neq V_{C}$ 的增量数据运行时间最短, 满足条件 $V_{d}^{\prime}=$ $V_{d}$ 且 $V_{C}^{\prime}=V_{C}$ 的增量数据运行时间最长, 原因是在 $V_{d}^{\prime} \neq V_{d}$ 且 $V_{C}^{\prime} \neq V_{C}$ 条件下进行增量数据更新时, 决 策类最优近似不变, 新增决策类的最优近似为新增 条件类, 整个过程中不需要新增计算; 而满足条件 $V_{d}^{\prime}=V_{d}$ 且 $V_{C}^{\prime}=V_{C}$ 的增量数据, 仅当决策类与新产 生的条件类相交为空集时才不用计算，最优近似保 持不变, 而相交为非空集时, 需根据不同情况进行 不同的计算，新产生的决策类也有类似过程，因 此, 总体来说所需时间最长. 新增其他两类数据的 过程有些类似，但是在讨论决策类分布变化时都会 比满足 $V_{d}^{\prime}=V_{d}$ 且 $V_{C}^{\prime}=V_{C}$ 时的运行时间要少, 所以 运行时间也是在上述两类之间.

图 2 为新增单个对象, 并在不同数据集上进行 增量更新与非增量更新时, 求解最优近似算法的运

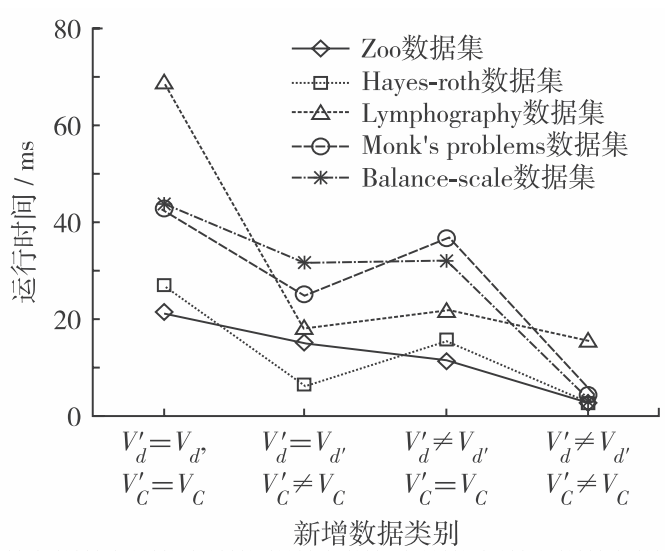

图 1 不同数据集在不同增量类别时的更新时间

Fig. 1 Update time for different categories of data on different data sets

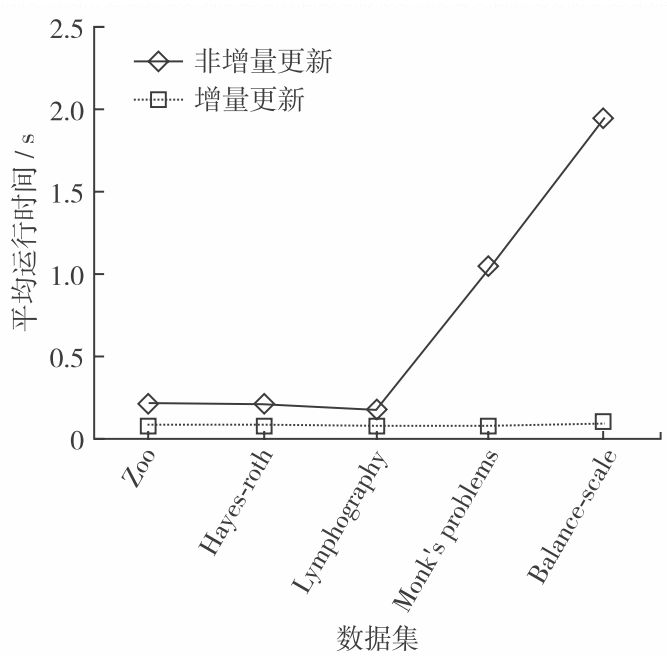

图 2 增量与非增量更新时间对比

Fig. 2 Comparison of incremental and non-incremental update time

行时间结果. 运行时间取 5 次随机新增一个对象时 的运行时间的平均值. 由图 2 可见，利用增量更新 与非增量更新方法，在不同数据集上计算最优近似 的耗时并不一样，增量更新方法所需时间较少，优 势明显. 当数据集决策类增加且数据集中的对象数 较多时, 增量更新方法的耗时也会增长, 但并不明 显, 而非增量算法的耗时却呈快速增长. 可见，增 量更新算法的时间效率要优于非增量更新, 究其原 因主要是在动态环境下, 当对象增加时, 采用非增 量更新方法计算最优近似集需要比较每个决策类与 每个条件类, 造成较多重复计算, 而对于增量更新 方法, 只需关注变化的条件类对决策类正域以及边 界类分布值的影响, 减少了比较次数, 提高了效 率. 尤其是当数据集较大时, 增量更新方法具有较 
好的时间性能，其计算最优近似集的时间效率明显 优于非增量方法计算.

\section{结 语}

针对决策系统动态变化情况下，决策类的最优 近似更新问题，本研究就单个对象增加引起值域发 生改变的 4 种不同情况，讨论了决策类的最优近似 的变化机制，并给出 4 种不同变化情形下的最优近 似更新求解方法. 仿真实验结果表明，所提增量式 更新算法能够快速地对最优近似结果进行快速更 新, 具有很高的计算性能.

基金项目: 国家自然科学基金资助项目（61991401); 江西省自然 科学基金重点资助项目 (20192ACBL20010)

作者简介：罗来鹏（1973-)，华东交通大学副教授. 研究方向： 粗糙集与粒计算. E-mail: luolp789@ 163.com

引文: 罗来鹏, 范自柱. 粗糙集最优近似的动态更新方法 $[\mathrm{J}]$. 深圳大学学报理工版, 2021，38(3): 324-330

\section{参考文献 / References:}

[ 1 ] PAWLAK Z. Rough sets [J]. Information and Computer Science, 1982, 11(5) : 341-356.

[ 2 ] 梁吉业, 李德玉. 信息系统中的不确定性与知识获取 $[\mathrm{M}]$. 北京: 科学出版社, 2005.

LIANG Jiye, LI Deyu. The uncertainty and knowledge acquiring in information systems $[\mathrm{M}]$. Beijing: Science Press, 2005. (in Chinese)

[ 3 ] ZIARKO W. Variable precision rough set model $[\mathrm{J}]$. Journal of Computer and System Science, 1993, 46(1): $39-59$.

[ 4 ] ZIARKO W. Probabilistic approach to rough sets $[\mathrm{J}]$. International Journal of Approximate Reasoning, 2008 , 49 (2) : 272-284.

[ 5 ] CHENG Yusheng, ZHANWenfa, WU Xindong. Automatic determination about precision parameter value based on inclusion degree with variable precision rough set model $[\mathrm{J}]$. Information Sciences, 2015, 290: 72-85.

[6]张清华, 薛玉斌, 胡 峰, 等. 粗䊁集近似集不确定 性研究 $[\mathrm{J}]$. 电子学报, 2016, 44(7)：1574-1580.

ZHANG Qinghua, XUE Yubin, HU Feng, et al. Research on uncertainty of approximation set of rough set $[\mathrm{J}]$. Acta Electronica Sinica, 2016, 44 (7) : 1574-1580. (in Chinese)

[ 7 ] 张清华, 薛玉斌, 王国胤. 粗䊁集的最优近似集 $[\mathrm{J}]$. 软件学报, 2016, 27(2): 295-308.

ZHANG Qinghua, XUE Yubin, WANG Guoyin. Optimal approximation sets of rough sets $[\mathrm{J}]$. Journal of Software, 2016, 27(2): 295-308. (in Chinese)

[8]罗来鹏，范自柱. 粗䊅集中几种粒结构的代数关系
$[J]$. 华侨大学学报自然科学版, 2019, 40(5)：694700 .

LUO Laipeng, FAN Zizhu. Algrbraic relation of several granular structures in rough set $[\mathrm{J}]$. Journal of Huaqiao University (Natural Science), 2019, 40 (5) : 694-700. (in Chinese)

[9] YU Jianhang, CHEN Minghao, XU Weihua. Dynamic computing rough approximations approach to time-evolving information granule interval-valued ordered information system [J]. Applied Soft Computing, 2017, 60: 1829.

[10 ] LI Yan, JIN Yongfei, SUN Xiaodian. Incremental method of updating approximations in DRSA under variations of multiple objects $[\mathrm{J}]$. International Journal of Machine Learning and Cybernetics, 2018, 9(2) : 295-308.

[11] CHENG Yi. Dynamic maintenance of approximations under fuzzy rough sets $[\mathrm{J}]$. International Journal of Machine Learning and Cybernetics, 2018, 9 ( 12 ): 2011-2026.

[12] HU Chengxiang, LIU Shixi, LIU Guoxiu. Matrix-based approaches for dynamic updating approximations in multigranulation rough sets $[\mathrm{J}]$. Knowledge-Based Systems, 2017, 122: 51-63.

[13 ] JING Yunge, LI Tianrui, FUJITA H, et al. Anincremental attribute reduction approach based on knowledge granularity with a multi-granulation view [ J ]. Information Sciences, 2017, 411(6) : 23-38.

[14] ZHANG Chucai, DAI Jianhua, CHEN Jiaolong. Knowledge granularity based incremental attribute reduction for incomplete decision systems $[\mathrm{J}]$. International Journal of Machine Learning and Cybernetics, 2020, 11 ( 5 ): 1141-1157.

[15] WEI Wei, SONG Peng, LIANG Jiye, et al. Accelerating incremental attribute reduction algorithm by compacting a decision table $[\mathrm{J}]$. International Journal of Machine Learning and Cybernetics, 2019, 10(9) : 2355-2373.

[16] GE Hao, LI Longshu, YANG Chuanjian, et al. Incremental reduction algorithm with acceleration strategy based on conflict region $[\mathrm{J}]$. Artificial Intelligence Review, 2019, 51(4) : 507-536.

[17] HU Jie, LI Tianrui, CHEN Hongmei, et al. An incremental learning approach for updating approximations in rough set model over dual universes $[\mathrm{J}]$. International Journal of Intelligent Systems, 2015, 30(8) : 923-947.

[18 ] LUO Chuan, LI Tianrui, CHEN Hongmei. Dynamic maintenance of approximations in set-valued ordered decision systems under the attribute generalization $[\mathrm{J}]$. Information Sciences, 2014, 257: 210-228.

【中文责编：英 子; 英文责编: 木 柯】 